\title{
Evaluation and further development of car following models in microscopic traffic simulation
}

\author{
P. Hidas \\ School of Civil and Environmental Engineering, \\ University of New South Wales, Sydney, Australia
}

\begin{abstract}
This paper presents findings from a comparative evaluation of car-following behaviour in a number of microscopic traffic simulators. The paper describes the results of the calibration and validation of the car following model implemented in the ARTEMiS simulator developed by the author. The calibration and validation are based on a 'standard' car-following experiment (called the Boschtest) which used instrumented vehicles to record the speed and relative distance between follower and leader vehicles on a one-lane road, and which was previously used to evaluate a number of other state-of-the-art simulators, including AIMSUN, PARAMICS and VISSIM. The simulated car following behaviour was compared to the field data using a number of error tests. A comparison of the results showed that the ARTEMiS car following model produced the closest match to the observed data out of all the tested models. In the last section of the paper, a number of traffic situations are identified where most car following models fail to reproduce real-life driver behaviour, and solutions to overcome these weaknesses are recommended for further development of car following models in microsimulation.

Keywords: microsimulation, car following, traffic modelling.
\end{abstract}

\section{Introduction}

Microscopic traffic simulation models are becoming increasingly important tools in modelling transport systems. They can be used to model complex transport networks and evaluate various traffic management alternatives in order to determine the optimum solution for traffic problems that cannot be studied by 
other analytical methods. While microscopic simulators are generally regarded as the best available tools for such purposes, several problems were also identified including computational performance, the accuracy of models in representing the traffic flow, and the difficulty of integration with advanced traffic management and traffic information systems. A topic of increasing concern is the validity of the microscopic sub-models, such as the car following and lane changing models. Car following behaviour, in particular, has a significant impact on the accuracy of the simulation model in replicating traffic behaviour on the road.

A previous paper [1] reported on the evaluation of the lane changing and merging abilities of several microsimulation models. This paper presents findings from a comparative evaluation of car-following behaviour in a number of microscopic traffic simulators. The paper describes the results of the calibration and validation of the car following model implemented in the ARTEMiS simulator developed by the author. The calibration and validation are based on a 'standard' car-following experiment (called the Bosch-test) which used instrumented vehicles to record the speed and relative distance between follower and leader vehicles on a one-lane road, and which was previously used to evaluate several other state-of-the-art simulators. The simulated car following behaviour are compared to the field data using a number of error tests, and conclusions and recommendations for further research and model development are presented at the last section of the paper.

\section{The field data used for the evaluation}

The greatest difficulty in the calibration and evaluation of car following models is obtaining field data in the required level of detail. One particular data set that is available for such purposes is what is called the "Bosch-test". The Robert Bosch GmbH Research Group [2] used an instrumented vehicle to record the difference in speed and headway between the instrumented vehicle and the vehicle immediately in front. The response of the follower vehicle (the instrumented vehicle), in terms of acceleration or deceleration, was also recorded. The data were recorded in $100 \mathrm{~ms}$ intervals for a total duration 300 seconds on a single lane road in Stuttgart, Germany. This dataset was made available to the research community and subsequently was used to evaluate a number of car following models [2,3]. Results of these previous evaluation studies provide a suitable benchmark for the evaluation of the car following model used in ARTEMIS.

\section{The ARTEMIS car following model}

The car-following model used in ARTEMIS was developed specifically for the microscopic simulation of urban interrupted traffic flow conditions. The model is based on the assumption that, when approaching and following a leader vehicle $(n-1)$ at any time $t$ the driver of the following vehicle $(n)$ attempts to adjust its acceleration so as to reach a desired spacing (D) after a time lag which takes $T$ 
seconds. Within the typical speed range of urban traffic ( 0 to $60 \mathrm{~km} / \mathrm{h}$ ) the desired spacing is assumed to be a linear function of the speed $(v)$ :

$$
D_{n}(t+T)=\alpha v_{n-1}(t+T)+\beta
$$

The parameters $\alpha$ and $\beta$ represent the desired spacing of driver $(n)$ and they are highly dependent on individual driver characteristics. As drivers are generally unable to achieve the exact desired spacing, a judgement error parameter $(\varepsilon)$ is used to account for the error of estimating the leader's movement characteristic. Thus, the condition can be described by the following equation:

$$
x_{n-1}(t+T)-x_{n}(t+T)=\varepsilon D_{n}(t+T)
$$

where $x_{i}(t)$ is the position of vehicle $i$ at time $t$. The judgement error parameter $\varepsilon$ is a random value with mean $=1.0$, variable in each time interval.

The proposed model eliminates the problems associated with the apparent reaction time inherent in previous models. The model has been validated for urban interrupted flow conditions and later extended to the higher (motorway) speed range. A full description of the car following model was published in [4].

\section{Model calibration}

For this evaluation study, the ARTEMIS car following model was implemented in a Microsoft Visual Basic function to allow easy analysis in Excel. As the model is based on a driver-dependent desired spacing criterion, it was necessary to calibrate the model to the characteristics of the particular driver that drove the instrumented car in the Bosch test. The first $240 \mathrm{~s}$ of the dataset were used for the calibration, while the last $60 \mathrm{~s}$ were used for the validation of the model. The desired spacing parameters, separately for acceleration, deceleration and highspeed driving range (see Table 1), were estimated using linear regression analysis in Excel. The values of $\mathrm{r}^{2}$ for the 3 parameter sets are indicating a reasonably high degree of correlation.

Table 1: $\quad$ The calibrated desired spacing parameters.

\begin{tabular}{|c|c|c|c|}
\hline \multirow{2}{*}{ Parameter } & \multicolumn{2}{|c|}{ Low speed } & \multirow{2}{*}{ High speed } \\
\cline { 2 - 3 } & Acceleration & Deceleration & \\
\hline$\alpha$ & 1.3717 & 1.698 & 4.0568 \\
\hline$\beta$ & 6.923 & 4.7836 & -21.66 \\
\hline$R^{2}$ & 0.56 & 0.65 & 0.59 \\
\hline
\end{tabular}


The judgment error parameter, $\varepsilon$, was calibrated by comparing the observed distance from the Bosch-test data with the modelled distance using the observed speed of the vehicle for each one second interval. The modelled distance values are estimated by linear regression in different speed groups. The mean value of $\varepsilon$ was confirmed as 1.0 and the standard deviation was found to be 0.19 . In the following model validation a random error parameter was drawn from a normal distribution using this calibrated mean and standard deviation range.

\section{Model validation}

The model was validated using the last $60 \mathrm{~s}$ of the Bosch-test data, which was not used in the calibration process. The car following model was used to calculate the acceleration, speed and position of the follower vehicle, using the speed and position of the leader vehicle and the calibrated model parameters. Figure 1 shows the observed and modelled trajectories for the validation time period. The two trajectories are very close, the differences can be attributed to the random variation in the judgement error parameter.

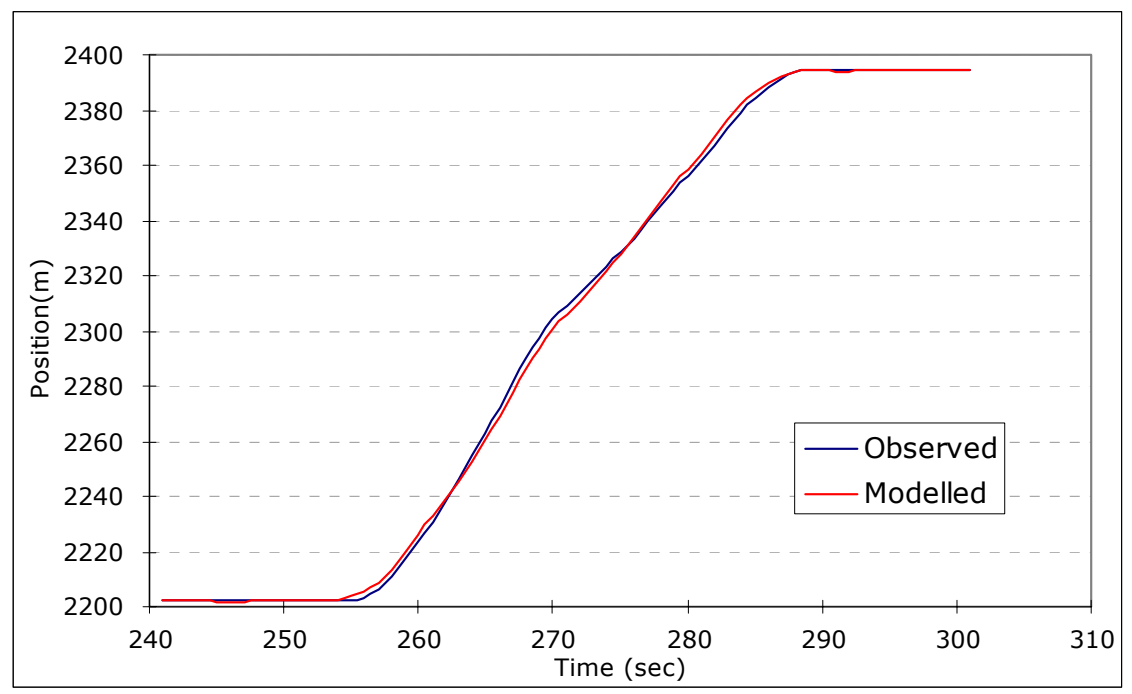

Figure 1: $\quad$ Comparison of observed and modelled trajectories.

Figure 2 shows a comparison of the observed and modelled spacing between the two vehicles. Again, there is a very close match between the two lines indicating a good fit of the model to the observed data, therefore the model is deemed to be a valid representation of the car following behaviour of the given driver. 


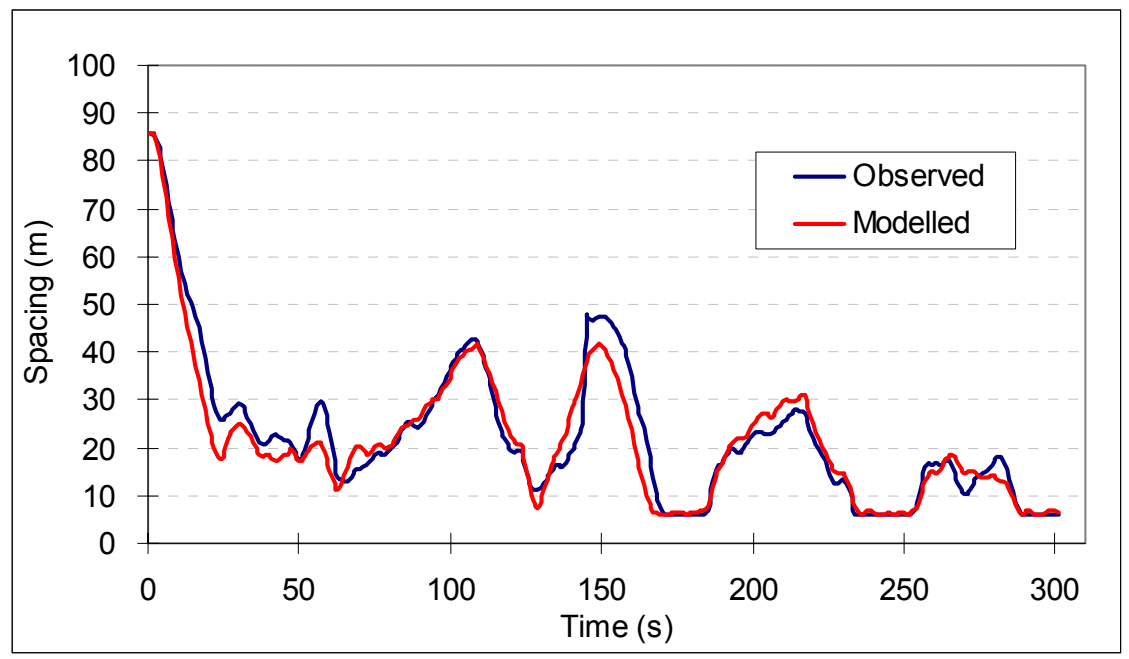

Figure 2: $\quad$ Comparison of observed and modelled spacing.

\section{Model evaluation}

Two error tests were used in this study to evaluate the performance of the car following model: the Root Mean Square (RMS) and Error Metric (EM) tests. The RMS error test measures the divergence of the modelled result from the observed value in the following form:

$$
\text { RMS Error }=\sqrt{\frac{1}{N} \sum_{n=1}^{N}\left(\frac{d_{s}}{d_{f}}\right)^{2}}
$$

where $d_{s}$ and $d_{f}$ are respectively the simulated and observed car following distances to the leader vehicle at simulation time $t$, and $N$ is the number of observations.

The Error Metric test is used to avoid over-rating discrepancies for large distances [2]. The EM is weighted by the logarithm of the ratio of modelled and observed distances and squared:

$$
\mathrm{EM}=\sqrt{\sum\left(\log \frac{d_{s}}{d_{f}}\right)^{2}}
$$

The above performance indicators were selected because the same statistics were used by other authors [2, 3, 5] to evaluate several other car following models. This allows a comparison of car following models across a number of simulators. Table 2 shows the performance measures of the car following models of several simulation models. 
Table 2: $\quad$ Comparison of car following models in selected simulators.

\begin{tabular}{|l|c|c|c|c|}
\hline $\begin{array}{l}\text { Model } \\
\text { (version) }\end{array}$ & AIMSUN & VISSIM & PARAMICS & ARTEMIS \\
\cline { 2 - 5 } & $\mathrm{v} 4.2$ & $\mathrm{v} 3.70$ & $\mathrm{v} 4.1$ & $\mathrm{v} 1.50$ \\
\hline Error Metric & 2.55 & 4.50 & 4.68 & $1.26-1.52$ \\
\hline RMS Error & 4.99 & 5.05 & 10.43 & $0.99-1.02$ \\
\hline
\end{tabular}

The values for the first three simulators in Table 2 are taken from a study by Panwai and Dia [5], while the last column shows the values from this evaluation. It must be noted that due to the use of the judgement error parameter, a random value in each time interval, in the ARTEMIS car following model, the modelled car following distances will be slightly different if the simulation is executed several times with the same input data. Therefore, to establish a range of performance measures that can be expected from ARTEMIS, the model was simulated a number of times, and the minimum-maximum values of the performance indicators were noted. These minimum-maximum values are indicated in Table 2 for the ARTEMIS model. It can be seen from Table 2 that the models replicate observed car following behaviour with varying degrees of accuracy, and ARTEMIS achieved a significantly closer fit to the observed data than the other models.
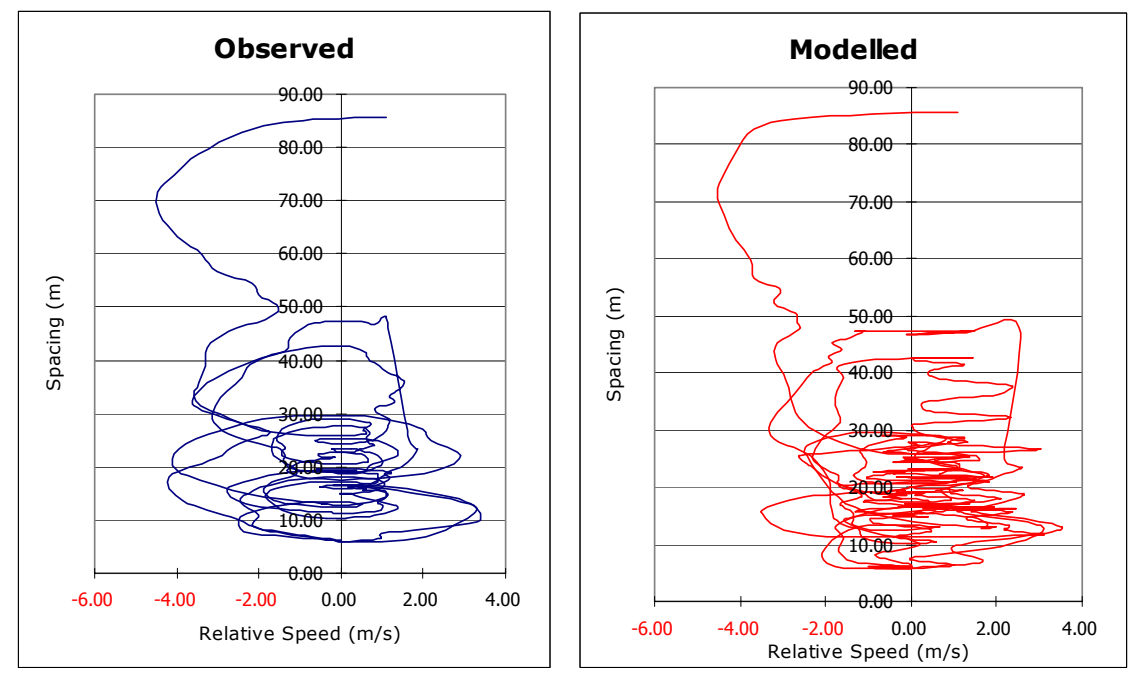

Figure 3: Comparison of observed and modelled drift behaviour.

Another measure of car following performance is a qualitative comparison of observed and modelled drift behaviour, as shown in Figure 3. It shows the oscillation (drift) of the spacing between the leader and follower vehicles as a function of the relative speed of the vehicles (i.e. leader speed minus follower speed). At the start of the test period the follower vehicle is far behind the leader, 
and the follower driver increases its speed (relative speed is negative) to catch up with the leader. When it gets close to the leader, the relative speed and, consequently, the spacing starts to oscillate around the desired spacing, because of the judgement error of the driver. Figure 3 shows a good qualitative match between the observed and modelled car following behaviour: while the curves representing the oscillations are obviously different, the range of modelled relative speed-and spacing-oscillation is very similar to the observed range.

These evaluation results show an excellent performance of the ARTEMIS car following model, in fact the statistical performance indicators for ARTEMIS are much better than for the other models. However, when interpreting these results, it is important to consider the following limitations of the Bosch-test used for the evaluation:

- the observed car following data in the Bosch-test relate to one follower driver only; it is obviously not appropriate to draw general conclusions from a one-person sample.

- the observed data covers only a 5-minute driving period, which is not enough to observe the full range of driver behaviour even for one driver. Studies have shown (for example [6]) that car following behaviour is highly variable over time.

- the observed data only covered "normal" driving situations on a single-lane roadway, and therefore it did not include any difficult or unusual conditions which may occur in everyday traffic. This test cannot provide any feedback on how the model would perform under such conditions.

\section{Specific problems in car following models}

The ARTEMIS car following model has now been in use for a long time in the microsimulation model. While the model performed well in most situations, several specific situations were identified where the car following model had weaknesses, requiring further improvements. This section summarises these problems and the improvements implemented in the model.

\subsection{Relationship with lane changing}

One general problem found was when a lane changing (or merging) occurs. In such situations, the vehicle moving in from another lane causes a distinct reduction of the spacing between its leader and follower vehicles. If the spacing before the lane change was close to the desired spacing at the given speed, it will be significantly less than the desired spacing, and as a result, the car following model would calculate an emergency deceleration, which in turn, would generate a shock-wave slowing down all the vehicles upstream in the lane. However, in real traffic, many lane changes occur at very short spacing without causing an emergency braking in the following vehicles. It seems that while drivers do have a given desired spacing (as a function of the speed), they are also willing to tolerate much shorter spacing under some circumstances: when they can clearly see the situation and can anticipate the actions of the other drivers. In the lane 
changing situation, instead of emergency braking, usually only a moderate/minimal deceleration is used to ensure that the spacing gradually increases to the desired spacing. According to observations, this process takes about 5-10 s.

This process creates an important link between the lane changing and car following models. One of the roles of the lane changing model is to determine under what conditions a vehicle is allowed to move into the target lane, and in this process it takes into account the spacing and speed of its potential leader and follower vehicles in the target lane. Thus, while the decision whether or not a lane change is "feasible" is based on the prevailing conditions in the target lane, at the same time it will have a great impact on the conditions after the lane change has taken place. This effect is now handled in ATREMiS by the improved car following model and the lane changing model described in [7]. The essence of the solution is a "relaxation period" of about $5 \mathrm{~s}$ (depending on the speed), during which the car following model calculates an acceleration which ensures a gradual increase of the spacing without emergency braking, until the normal desired spacing is restored between the vehicles.

\subsection{Queue discharge at signalised intersections}

Another situation where significant differences were found between the modelled and observed traffic conditions is queue discharge at the stop-line of signalised intersections. Many studies have shown that the saturation flow rates at signalised intersections are quite different at different sites and they can be as high as 2,150 veh/hour. However, the desired spacing clearly determines the average saturation flow rate, and the maximum saturation flow rate defined by the calibrated desired spacing parameters in this case would be only $1,744 \mathrm{veh} /$ hour. It is obvious then that drivers have a distinct different behaviour when starting at a signalised intersection stop-line. It seems logical to assume that due to the time constraints of the available green light, for a short period of time drivers are willing to take a somewhat higher level of risk and travel at closer spacing than under "normal" driving conditions while passing the stopline; once they get through the intersection, they gradually recover their desired spacing and the dense queue starts to break up. This effect is now modelled in ARTEMIS by allowing the user to define location-specific saturation flows for each intersection movement, and calculating the spacing parameters from the saturation flow accordingly when vehicles are approaching a signalised intersection at green signal. The model has shown satisfactory performance under several case studies, however, further research is needed to investigate the spatial extent of the queue discharge adjustment under real-life traffic conditions.

\section{Conclusions}

This paper presented the findings of a comparative evaluation of the ARTEMIS car-following model developed by the author. The model was calibrated and validated using a 'standard' car-following experiment, the Bosch-test, which was 
previously used to evaluate a number of other state-of-the-art simulators, including AIMSUN, PARAMICS and VISSIM. The simulated car following behaviour was compared to the field data using the Error Metric (EM) and Root Mean Square Error (RMSE) tests. A comparison of the results showed that the ARTEMiS car following model produced the closest match to the observed data out of all the tested models.

The Bosch test, using data from just one driver, showed that the ARTEMiS model can replicate the driver's car following behaviour more accurately than the other models. This is because the model was calibrated specifically to that particular driver. This confirms the assumption of the model, that car following is a highly individual behaviour, and, as generally accepted, there are significant differences among individuals.

Two specific driving situations were also identified where car following behaviour shows significant deviation from the "standard" behaviour that can be described by the desired spacing parameters. These are the transitional time period immediately following a lane change, and the queue discharge process at the approach to signalised intersections. Specific solutions were developed and implemented for these situations.

From this experiment, one might conclude that the ARTEMiS model is significantly more accurate than the other car following models used in microsimulation models. However, this is not necessarily the case, because when a larger driver population is simulated, it is not possible to calibrate the model to each individual driver. Rather, the model parameters can only be calibrated to the "average" behaviour and the variation between individual drivers can be represented by a range of parameters. In this process, the ARTEMiS model would likely to lose its advantage over the other models, and it is likely that its error level for the whole driver population would be closer to those obtained from the other models. The average error, calculated from modelling a larger driver population, would be more representative of the practical accuracy of the models - however, such a test would require much larger measured car following data sets that are not currently available. Nevertheless, the results of this study confirmed the abilities of the ARTEMIS car following model to represent individual car following behaviour in a realistic manner.

\section{Acknowledgements}

The evaluation study of the ARTEMIS car following model described in this paper was carried out by Feng Liu, as part of his Master of Engineering Science project at the University of New South Wales under the supervision of the author. However, the author is fully responsible for the conclusions of the study.

\section{References}

[1] Hidas, P., Lane changing and merging under congested conditions in traffic simulation models. Urban Transport XI - Urban Transport and the Environment in the 21st Century (Ed. C.A. Brebbia and L.C. Wadhwa), pp. 779-788, WIT Press, Southampton, Boston, 2005. 
[2] Manstetten, D., Krautter, W., and Schwab, T. "Traffic simulation supporting urban control system development." 4th World Congress on Intelligent Transport System, 1997.

[3] Barceló, J., and Casas, J. "Dynamic Network Simulation With AIMSUN." International Symposium on Transport Simulation, Yokohama, 2002.

[4] Hidas, P. A car following model for urban traffic simulation. Traffic Engineering + Control, Vol. 39 No 5, pp. 300-305, 1998.

[5] S. Panwai and H. Dia, "Comparative Evaluation of Car Following Models," IEEE Trans. Intelligent Transportation System, vol. 6, no. 3, pp. 314-325, 2005.

[6] Hidas, $\mathrm{P}$ and Wagner, $\mathrm{P}$ "Review Of Data Collection Methods For Microscopic Traffic Simulation", Proceedings of the 10th World Conference on Transport Research, (published on CD-ROM, no page numbers), Istanbul, Turkey, July 2004.

[7] Hidas, P "Modelling Vehicle Interactions in Microscopic Simulation of Merging and Weaving", Transportation Research Part C: Emerging Technologies, Volume 10, pp. 37-62. Elsevier Science Ltd, 2005. 